\title{
COMPLICACIONES POSTQUIRÚRGICAS DE COLECISTECTOMÍA LAPAROSCÓPICA, HOSPITAL TRAUMATOLÓGICO DR. NEY ARIAS LORA, JUNIO 2018-JUNIO 2019
}

\author{
Postoperative complications of laparoscopic cholecystectomy, \\ Traumatologic Hospital Ney Arias Lora, june 2018-june 2019
}

\section{Adia Victoria Henríquez Jiméneza ${ }^{a}$ Raybelin Peña Rodríguez ${ }^{b}, M_{a r y}$ Elissanny Peña Lorenzoc, Younine Hernández Castillo ${ }^{d}$, Yordano Montesano Jiménez ${ }^{e}$, Jorge Octavio Sánchez Orbe y Victoria de los Ángeles Ibarra Lesmag}

Recibido: 8 de diciembre, $2019 \bullet$ Aprobado: 3 de marzo, 2020

Cómo citar: Henríquez Jiménez AV, Peña Rodríguez R, Peña Lorenzo ME, Hernández Castillo Y, Montesano Jiménez Y, Sánchez Orbe JO, Ibarra Lesma V de los Ángeles. Complicaciones postquirúrgicas de colecistectomía laparoscópica, Hospital Traumatológico, Dr. Ney Arias Lora, junio 2018-junio 2019. cysa [Internet]. 17 de septiembre de 2020 [citado 18 de septiembre de 2020];4(3):115-121. Disponible en: https://revistas.intec.edu.do/index.php/cisa/article/view/1909

\section{Resumen}

Objetivo: determinar la frecuencia y causas de complicaciones postquirúrgicas de colecistectomía laparoscópica, en el Hospital Traumatológico Doctor Ney Arias Lora, junio 2018 - junio 2019.

Método: se realizó un estudio de tipo observacional descriptivo, de corte transversal

y de fuente retrospectiva, cuya población fue de 617 pacientes, de los cuales solo 19 presentaron complicaciones, representando la muestra de este estudio. Los datos fueron procesados utilizando el sistema Epi-Info 7.0.

Resultados: de 617 pacientes en cuales se realizó una colecistectomía laparoscópica, 19 presentaron complicaciones, para una tasa de $3.1 \%$; el estudio demostró que el sexo más afectado es el femenino entre los y 26-30 años.

\footnotetext{
a UNAP Guanito, Elias Piña, República Dominicana. Correo-e: avictoriahenriquez@gmail.com

b CPN Los Anones, San José de Ocoa, República Dominicana. Correo-e: raybelin18@gmail.com

${ }^{\mathrm{c}}$ UNAP Carreras de Yegua, San Juan, República Dominicana.

Correo-e: mary12lorenzo@gmail.com
}

\begin{abstract}
Objective: Determine the frequency and different causes of postoperative complications of laparoscopic cholecystectomy, in the Hospital Traumatológico Doctor Ney Arias Lora, june 2018-june 2019.

Methods: The study was descriptive, cross-sectional and retrospective with a population of 617 patients but only 19 showed postoperative complications, which was the sample number we use. The data was processed using Epi-Info 7.0.
\end{abstract}

Results: Of the 617 laparoscopic cholecystectomies perform in the hospital, 19 presented with postoperative complications, which put the postoperative complication rate at $3.1 \%$; the study showed that the feminine sex was the most affected and the most common age range was between 26-30 years old.

\footnotetext{
d Estudiante de medicina. República Dominicana.

Correo-e: youninehernandezcastillo@gmail.com

e Correo-e: yordano@foundationforpeace.org

${ }^{\mathrm{f}}$ Cruz Roja Dominicana, República Dominicana.

Correo-e: drsanchezorbe@gmail.com

${ }^{g}$ Estudiante medicina UNPHU, República Dominicana.

Correo-e: vikyibarra@gmail.com
} 
El tipo de complicación postquirúrgica más frecuente fueron las hemorragias causando un $33 \%$ de las complicaciones, seguidas por náuseas y/o vómitos con un $21 \%$ de las mismas.

Conclusión: el estudio demuestra que, dentro de las complicaciones principales de colecistectomías laparoscópicas, las hemorragias ocupan el primer lugar, representando un $33 \%$ de las complicaciones, seguidas por un $21 \%$ de náuseas y/o vómitos y, en tercer lugar, la ictericia y la omoalgia con $17 \%$ cada una.

Palabras clave: colecistectomía; laparoscopia; complicaciones; hemorragia; frecuencia.

\section{Introducción}

Las enfermedades gastrointestinales representan una gran molestia para los pacientes que la padecen, y a la vez, un gran peso para sus familiares y para el Estado. Uno de los grupos patológicos más comunes lo constituyen las enfermedades de las vías biliares, que, con el paso de los años y la modificación de la dieta y el estilo de vida de las generaciones, se ha acrecentado. ${ }^{1-3}$

Con este estudio se buscó establecer un punto de comparación estadístico de complicaciones de este procedimiento entre los datos nacionales y los niveles de incidencia detallados en las literaturas internacionales, logrando evidenciar que coincidía con los estudios de América Latina en cuanto a las incidencias de complicaciones.

En el año 2015, en la ciudad de Guayaquil, Ecuador, se realizó un estudio por el doctor Luis Daniel Benítes Delgado, titulado Complicaciones postcolecistectomía laparoscópica en el Hospital IESS Milagro, periodo 2014-2015, se concluyó que la mayor complicación fue la lesión de vías biliares con $26 \%$ continuado por las lesiones por trocar y aguja de Veress con $18 \%$, hemorragia $14 \%$, dolor postoperatorio $13 \%$, hemoperitoneo $11 \%$,
The most common postoperative complication was hemorrhages, representing $33 \%$ of all the complications, follow by nausea and/or vomits with $21 \%$ of the complications.

Conclusion: The study shows that within the main postoperative complications of laparoscopic cholecystectomies, the hemorrhages constituted the \#1 cause with a $33 \%$, meanwhile nausea and/or vomits represented the $21 \%$ and in third place there was a tide between jaundice and shoulder pain with $17 \%$.

Keywords: Cholecystectomy; laparoscopy; complications; hemorrhages; frequency.

infección de herida quirúrgica $9 \%$ y colecciones intraabdominales $9 \%{ }^{4}$

En la ciudad de Lima, Perú, en el 2017, se realizó una investigación titulada Factores asociados a complicaciones postoperatorias en pacientes adultos mayores sometidos a colecistectomía laparoscópica en el Hospital Vitarte 2012- 2015 por la Dra. Melissa Flor Salirrosa Sepúlveda. Como resultado se obtuvo que la edad media fue 67.4 años, siendo la edad mínima 60 y la máxima 89 años, distribuidos según el sexo un $73,2 \%$ representado por las mujeres y en un 26,8 $\%$ por los hombres y se concluyó que dentro de las características epidemiológicas asociadas a complicaciones en pacientes mayores sometidos a este tipo de procedimiento quirúrgico están: mayor de 70 ańos, el sexo femenino, obesidad y haber tenido una cirugía previa. ${ }^{5}$

En Santo Domingo, República Dominicana, en el 2018, el Dr. Robert Romero Jerez realizó una investigación intitulada Cuáles son las complicaciones de la colecistectomia laparoscópica electiva en el Hospital Salvador Bienvenido Gautier en el periodo noviembre 2017-abril 2018, “el universo se constituyó por 150 paciente, siendo incluidos en la investigación solo 19. De estos 19 el $88 \%$ de los pacientes presentaron complicaciones. De las complicaciones en el 
transoperatorio se observó en un 83.3 \% lesión de víscera sólida, donde el hígado fue el más afectado; y de las complicaciones postquirúrgicas el $55 \%$ padeció de infección del área quirúrgica. ${ }^{6}$

\section{Materiales y métodos}

Se realizó un estudio de tipo observacional descriptivo, de corte transversal y de fuente retrospectiva de información, para determinar la frecuencia de complicaciones postquirúrgicas de colecistectomía laparoscópica en el Hospital Traumatológico Doctor Ney Arias Lora, durante el período de junio 2018-junio 2019.

La muestra estuvo conformada por todos los pacientes que presentaron complicaciones postquirúrgicas de colecistectomía laparoscópica, un total de 19, los cuales se determinaron a través de un muestreo no probabilístico por conveniencia o intencional, cuyo criterio de inclusión fue todos los pacientes con complicaciones de colecistectomía laparoscópica que hayan sido atendidos en el Hospital Traumatológico Doctor Ney Arias Lora en el periodo de junio 2018 a junio 2019. Se excluyeron todos los pacientes que no cumplieran con el requisito de inclusión.

La información utilizada fue recolectada de los expedientes clínicos de los pacientes, facilitados por la unidad de Archivo Clínico del Hospital Traumatológico Doctor Ney Arias Lora.

Se utilizó como instrumento de recolección de datos una encuesta tipo, consistente en 10 preguntas cerradas, de las cuales 8 eran independientes y 2 dependientes de las respuestas registradas como afirmativas en el cuestionario.

\section{Resultados}

En este estudio se determinó que, del total de los pacientes estudiados, un $89 \%$ pertenecen al sexo femenino mientras que el $11 \%$ restante correspondieron al sexo masculino.
Gráfico 1. Distribución según género pacientes $n=$ 19. Hospital Traumatológico Doctor Ney Arias Lora, junio 2018-junio 2019

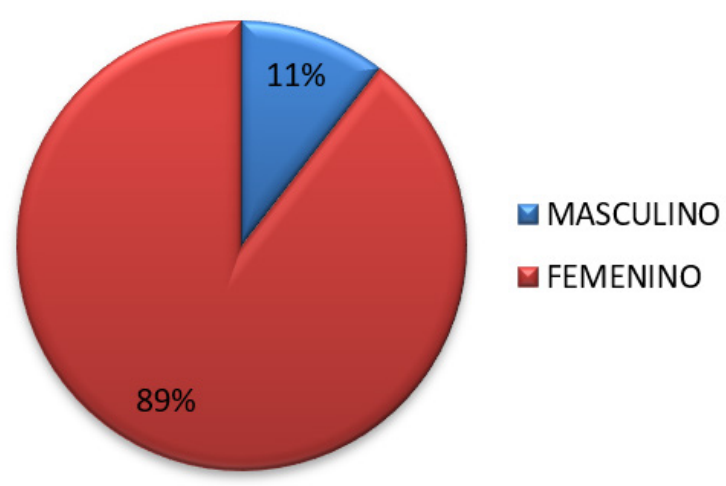

Fuente: formulario para la recolección de datos de los expedientes del departamento de Archivo Clínico del Hospital Traumatológico Doctor Ney Arias Lora.

El grupo de edad más afectado fue el de pacientes entre 26-35 ańos de edad, siendo estos un $32 \%$ de los casos, seguidos por los pacientes con edades comprendidas entre los 36-45 ańos de edad, representando el $21 \%$ de los casos conjuntamente con el grupo de edad entre 46-55 años, que de igual manera representan un $21 \%$ de los casos afectados.

Gráfico 2. Distribución según edad pacientes $n=$ 19. Hospital Traumatológico Doctor Ney Arias Lora, junio 2018-junio 2019

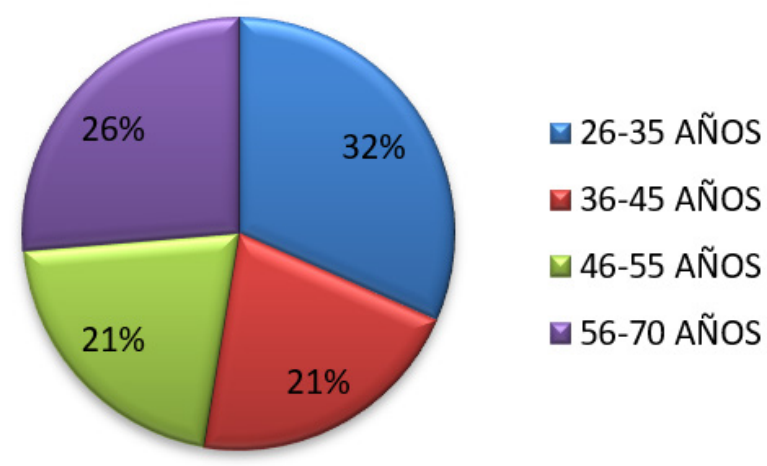

Fuente: formulario para la recolección de datos de los expedientes del departamento de Archivo Clínico del Hospital Traumatológico Doctor Ney Arias Lora. 
De las diferentes patologías biliares que pueden terminar en una colecistectomía laparoscópica, en nuestro estudio pudimos observar que el $100 \%$ fue secundario a una colelitiasis.

Tabla 1. Frecuencia de complicaciones postquirúrgicas. Hospital Traumatológico Doctor Ney Arias Lora, junio 2018-junio 2019

\begin{tabular}{|l|l|l|}
\hline $\begin{array}{l}\text { PATOLOGÍA } \\
\text { BILIAR }\end{array}$ & FRECUENCIA & PORCENTAJE \\
\hline COLELITIASIS & 19 & 100.00 \\
\hline $\begin{array}{l}\text { PÓLIPOS } \\
\text { VESICULARES }\end{array}$ & 0 & 0.00 \\
\hline $\begin{array}{l}\text { CÁNCER DE } \\
\text { VESÍCULA }\end{array}$ & 0 & 0.00 \\
\hline TOTAL & 19 & 100.00 \\
\hline
\end{tabular}

Fuente: formulario para la recolección de datos de los expedientes del departamento de Archivo Clínico del Hospital Traumatológico Doctor Ney Arias Lora.

De los pacientes que presentaron complicaciones postquirúrgicas de una colecistectomía laparoscópica, $21 \%$ tenían hipertensión arterial como comorbilidad al momento de la cirugía mientras que un $5 \%$ tenían diabetes mellitus como antecedente patológico.

El $100 \%$ de las colecistectomías laparoscópicas fueron de forma electiva, es decir, que fueron preparadas con anterioridad.

Tabla 2. Frecuencia programación postquirúrgica. Hospital Traumatológico Doctor Ney Arias Lora, junio 2018-junio 2019

\begin{tabular}{|l|l|l|}
\hline PROGRAMACIÓN & FRECUENCIA & PORCENTAJE \\
\hline ELECTIVA & 19 & 100.00 \\
\hline EMERGENCIA & 0 & 0.00 \\
\hline TOTAL & 19 & 100.00 \\
\hline
\end{tabular}

Fuente: formulario para la recolección de datos de los expedientes del departamento de Archivo Clínico del Hospital Traumatológico Doctor Ney Arias Lora.
Dentro de las complicaciones de las colecistectomías laparoscópicas, este estudio estableció que las comunes fueron las hemorragias, constituyendo un $33 \%$ de las mismas, mientras que las náuseas y/o vómitos correspondieron a un $21 \%$ de las complicaciones, y en tercer lugar se encontraron la ictericia y la omoalgia con un $17 \%$ para ambas sin embargo la complicación menos frecuente fue la lesión de víscera hueca presentándose en un $4 \%$ de los casos.

Grafico 3. Distribución según complicaciones clínicas postquirúrgicas. Hospital Traumatológico Doctor Ney Arias Lora, junio 2018-junio 2019

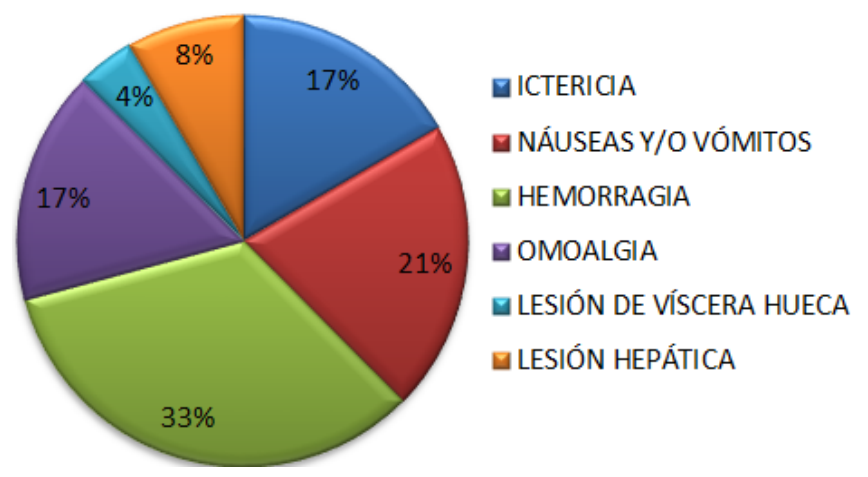

Fuente: formulario para la recolección de datos de los expedientes del departamento de Archivo Clínico del Hospital Traumatológico Doctor Ney Arias Lora.

Igualmente se determinó el tiempo de estadía postquirúrgica de los pacientes, cuyo resultado arrojo que $32 \%$ se mantuvo hospitalizado por dos días, mientras que un $5 \%$ se mantuvo hospitalizado por más de siete días.

\section{Discusión}

La colecistectomía laparoscópica es uno de los procedimientos quirúrgicos realizados con más frecuencia a nivel mundial en el campo de la cirugía general, esta técnica se caracteriza por cursar con baja morbilidad y con las ventajas ampliamente conocidas de la cirugía mínimamente invasiva. 
La colecistectomía laparoscópica tiene la peculiaridad de ser un procedimiento seguro, efectivo y reproducible, debe ser considerada como la cirugía ideal al momento de cualquier patología cística que requiera extirpación de la vesícula biliar. ${ }^{7-9}$

A pesar de ello, como todo procedimiento invasivo, puede presentar complicaciones después de la cirugía, las cuales tienden a ser poco frecuentes, pero pueden llegar a ser graves para el paciente. ${ }^{10}$

Según el estudio realizado de los 617 pacientes que se intervinieron vía laparoscópica para colecistectomía, solo se complicaron 19, para un porcentaje de $3.1 \%$, no coincidiendo con el estudio realizado por Fung et al., en el Hospital de Vitarte, en donde queda en evidencia una mayor tasa, con un porcentaje de $8.2 \% .^{11}$

Por otra parte, en el estudio realizado por Prieto et al., en el Hospital General Luis Vernaza, se mostró una incidencia de complicaciones de colecistectomía laparoscópica, presentó una tasa de $18.3 \%{ }^{11}$

El sexo femenino fue el más frecuente (89\%), mientras que el sexo masculino fue el de menos presentación (11\%), coincidiendo con el estudio realizado por Fung et al., en el Hospital de Vitarte, en donde queda en evidencia una mayor frecuencia en el sexo femenino (64\%). ${ }^{12}$ Los datos arrojados por la investigación realizada por el Dr. Robert Romero et al., realizada en el Hospital Salvador Bienvenido Gautier, concuerdan con lo anterior, el sexo femenino fue más frecuente $(83.3 \%)$, en comparación con el sexo masculino $(16.7 \%) .^{13}$

El rango de edad más frecuente, según la presente investigación fue de 26-35 años de edad (32\%), que se corresponde con el tercer y cuarto decenio de vida, mientras que los rangos de edad de menor frecuencia fueron los de 36-45 años y 46-55 años $(21 \%)$, aproximándose un poco al estudio realizado por Prieto et al., en el Hospital General Luis
Vernaza, en el cual el rango de edad más frecuente fue de 30 a 44 años (30.9\% ) 11 y en total diferencia con respecto al estudio realizado por Fung et al., en el Hospital de Vitarte, en el cual el rango de edad más frecuente fue de 50-60 años (44\%). ${ }^{14}$

Según el diagnóstico preoperatorio todas las intervenciones que se realizaron en los pacientes del estudio fueron a causa de una colecistitis por colelitiasis (100 \%), siendo esta la causa principal. En el estudio realizado por Murriagui las cifras fueron casi similares, siendo el diagnóstico preoperatorio más frecuente la colecistitis por colelitiasis (93.4\%), y el menos frecuente la colecistitis aguda $(6.6 \%) .{ }^{15}$

Con respecto a la parte de la programación quirúrgica, todas las colecistectomías laparoscópicas realizadas en los pacientes investigados fueron electivas $(100 \%)$, lo que quiere decir que fueron preparadas con anterioridad.

En relación con las complicaciones postquirúrgicas de colecistectomía laparoscópica según la investigación realizada presenta que la hemorragia (33 $\%$ ) fue la complicación más frecuente y la lesión de víscera hueca (4\%) la menos frecuente. Siendo estos resultados semejantes a los obtenidos en el estudio realizado por Fung et al., en el Hospital de Vitarte, en el cual las hemorragias (89 \%) son las complicaciones propias de la colecistectomía más frecuente. ${ }^{11} \mathrm{~A}$ diferencia del estudio de Murriagui, en el cual los vómitos fueron la complicación predominante. ${ }^{15}$

La estancia hospitalaria de la complicación postquirúrgica está determinada por varios factores. Los resultados arrojados por el estudio realizado demuestran que el mayor porcentaje de los pacientes tuvieron una estancia hospitalaria de dos días (32\%) y el menor porcentaje quedó representado por los pacientes con siete o más días (1\%) de estancia en el hospital. Este resultado es similar al que obtuvo Rubén et al., realizado en el Hospital General de 
Boca del Río, en el cual también el mayor porcentaje de los pacientes tuvieron una estancia en el hospital de dos días $(52 \%)$, después le seguían los que duraron menos de 24 horas $(36 \%)$ y, por último, los que estuvieron tres días (10\%) de estancia en el hospital. ${ }^{16}$

\section{Conclusiones}

De un total de 617 pacientes con el diagnóstico de colelitiasis en el Departamento de cirugía general en el Hospital Traumatológico Doctor Ney Arias Lora en el periodo junio 2018-junio 2019 se pudo observar lo siguiente:

- Diecinueve (19) pacientes presentaron complicaciones, para una tasa de $3.1 \%$.

- Predominó el sexo femenino representando el $89 \%$ de los casos.

- Las edades más afectadas fueron entre 26-30 años correspondientes a un $32 \%$ del total.

- La causa principal, según el diagnóstico, fue colecistitis por colelitiasis, siendo esta la única y total causa de la misma.

- Las comorbilidades más frecuentes fue hipertensión arterial representando un $21 \%$ y en menor medida la diabetes mellitus con un $5 \%$.

- El tipo de complicación postquirúrgica más frecuente fue la hemorragia, correspondiendo al $33 \%$ de los casos seguido por las náuseas y/o vómitos representando el $21 \%$ de los casos y, en menor medida, las lesiones de víscera hueca con un $4 \%$.

- Las colecistectomías laparoscópicas fueron electivas en su totalidad.

- El tiempo de estancia hospitalaria más frecuente por complicación postquirúrgica fue de dos días.

\section{Bibliografía}

1. Schwartz S, Brunicardi F, Andersen D. Principios de cirugía. (9th ed.). México, D.F.: McGraw-Hill Interamericana; 2015.

2. Intramed. Vías y vesícula biliares: embriología, anatomía y fisiología. Intramed.net. 2019. [Online]. Available from: https://www. intramed.net/sitios/librovirtual8/pdf/8_05.pdf [Accessed 3rd July 2019].

3. Hall J, Guyton A. Guyton and Hall textbook of medical physiology. (23rd ed.). Philadelphia: Elsevier; 2016.

4. Benítes Delgado L. (2015). Complicaciones post colecistectomía laparoscópica en el Hospital IESS Milagro, periodo 2014-2015 [Tesis doctoral]. Universidad de Guayaquil, Guayaquil, Ecuador.

5. Salirrosa Sepúlveda M. Factores asociados a complicaciones postoperatorias en pacientes adultos mayores sometidos a colecistectomía laparoscópica en el Hospital Vitarte 2012- 2015. (Tesis de especialidad. Universidad Ricardo Palma) Recuperado de: http://repositorio.urp.edu.pe/ bitstream/handle/urp/1152/TESIS\%20-\% 20LIZETH\%20ROSSELLY\%20FERN\%C3\%81NDEZ\%20CAUTI\%20HECHO. pdf?sequence $=1 \&$ isAllowed $=y$

6. Romero Jerez J. Cuáles son las complicaciones de la colecistectomía laparoscópica electiva en el Hospital Salvador Bienvenido Gautier en el periodo noviembre 2017-abril2018. (Tesis de especialidad. Universidad Nacional Pedro Henríquez Ureña). Recuperado de: https://repositorio.unphu. edu.do/bitstream/handle/123456789/1311/ Cua\%CC\%81les\%20son\%20las\%20complicaciones $\% 20$ de $\% 201 \mathrm{la} \% 20$ colecistectom $\%$ C3\%ADa\%20\%20laparosco\%CC\%81 pica\%20 electiva $\% 20$ en $\% 20$ el $\% 20$ Hospital $\% 20$ Salvador\%20Bienvenido\%20Gautier\%20 en $\% 20$ el\%20periodo $\% 20$ noviembre\%202017abril\%202018.pdf?sequence $=1$ \&isAllowed $=y$ 
7. Prieto Álvarez R. (2015). Complicaciones y estancia hospitalaria de mujeres entre 15 y 90 años, sometidas a colecistectomía laparoscópica Hospital Luis Vernaza año 2014-2015. (Tesis de grado, Universidad de Guayaquil). Recuperado de: http://repositorio.ug.edu.ec/handle/redug/10885.

8. MedlinePlus en español: Enfermedades de la vesícula biliar. [Online]. Available from: https://medlineplus.gov/spanish/gallbladderdiseases.html [Accessed 14th June 2019].

9. Vicente López E, Quijano Collazo Y. Cirugía Laparoscópica: Aspectos Generales [Online]. Available from: http://www.cirugiasanchinarro.com/site s/default/files/cirugia_laparoscopica.pdf [Accessed 14th June 2019].

10. Limaylla-Vega H, Vega-Gonzales E. (2017). Lesiones iatrogénicas de las vías biliares. [Online]. Recuperado de: http://www. scielo.org.pe/scielo.php?script $=$ sci_arttex$\mathrm{t} \&$ pid $=S 102251292017000400010$ [Accessed 20th Jul. 2019].

11. Jara G, Rosciano J, Barrios W. Colecistectomía laparoscópica subtotal como alternativa quirúrgica segura en casos complejos [Online]. ELSEVIER. 2017. Available from: https:// www.elsevier.es/es-revistacirugiaespanola36articulocolecistectomia-laparoscopica-subtotal-como-alternativa-S0009739X17301768 [Accessed 25th July 2019].

12. Kim S, Donahue T. Colecistectomía laparoscópica [Online]. Available from: https://sites. jamanetwork.com/spanish-patient-pages/2018/ hoja-para-el-paciente-de-jama-180501.pdf [Accessed 25th July 2019].
13. Fung Arroyo de los Santos R. Frecuencia de las complicaciones de la colecistectomía laparoscópica en el hospital de vitarte durante el periodo del 2015. (Tesis de especialidad, Universidad Rircardo Palma) Recuperado de:http://repositorio.upsjb.edu.pe/handle/upsjb/1133

14. Romero Jerez R. Cuáles son las complicaciones de la colecistectomía laparoscópica electiva en el Hospital Salvador Bienvenido Gautier en el periodo noviembre 2017-abril 2018.(Tesis de especialidad, Universidad Pedro Henríquez Ureña).Recuperado de: http://repositorio.unphu.edu.do/bitstream/ handle/123456789/1311/Cuáles\%20son\%20 las\%20complicaciones\%20de\%20la\%20colecistectomía\%20\%20laparoscópica\%20electiva\%20 en $\% 20$ el $\% 20$ Hospital\%20Salvador\%20Bienvenido\%20Gautier\%20en\%20el\%20periodo\%20 noviembre\%202017abril\%202018.pdf?sequence $=1$ \&isAllowed $=y$ [Accessed 25th July 2019].

15. Barrera M, Margoth E. Complicaciones postquirúrgicas de la colecistectomía laparoscópica, en pacientes atendidos en el área de cirugía general del Hospital Alfredo Noboa Montenegro. Recuperado de: http://dspace.uniandes. edu.ec/bitstream/123456789/9254/1/ PIUAMED055-2018.pdf

16. Cordero García R, Pérez Santos K, García Rodríguez A. Tiempo de estancia hospitalaria post quirúrgica en pacientes post operados de Colecistectomía convencional y laparoscópica [Internet]. Pdfs.semanticscholar.org. 2015 [cited 26 July 2019]. Recuperado de: https:// pdfs.semanticscholar.org/c559/e9fa4d9df88f2a50c853d1b2064921b8eab8.pdf 